\title{
Declarative Data Generation with ProbLog
}

\author{
Anton Dries \\ KU Leuven, Dept. of Computer Science \\ Celestijnenlaan 200A - bus 2402 \\ 3001 Heverlee, Belgium \\ anton.dries@cs.kuleuven.be
}

\begin{abstract}
In this paper we describe a novel declarative approach to data generation based on probabilistic logic programming. We show that many data generation tasks can be described as a probabilistic logic program. To this end, we extend the ProbLog language with continuous distributions and we develop a simple sampling algorithm for this language. We demonstrate that many data generation tasks can be described as a model in this language and we provide examples of generators for attribute-value data, sequences, graphs and logical interpretations and we show how to model common extensions such as noise, missing values and concept drift.
\end{abstract}

\section{INTRODUCTION}

Machine learning is an experimental science in a sense that many of its results are validated in an empirical way. As in any other science, the goal of experimentation is twofold: (1) to validate the developed methodology or hypothesis, and (2) to study the behavior of a system in varying conditions in order to gain a deeper understanding.

On the importance of experimental design for machine learning, Pat Langley wrote the following [25]:

To study the effect of 'nurture' on a learning system, one must vary the environment or domain in which it learns. Natural domains, [...], are the most obvious because they show real-world relevance. Also, successful runs on a number of different natural domains provide evidence of generality. However, such environments give little aid in understanding the effects of domain characteristics on learning, since they do not let one independently vary different aspects of the environment. For this, experiments with artificial domains are essential.

In addition to real data, artificial data is of vital importance to achieve a better understanding of the algorithms and methodologies of machine learning. In contrast to real-

Permission to make digital or hard copies of all or part of this work for personal or classroom use is granted without fee provided that copies are not made or distributed for profit or commercial advantage and that copies bear this notice and the full citation on the first page. Copyrights for components of this work owned by others than the author(s) must be honored. Abstracting with credit is permitted. To copy otherwise, or republish, to post on servers or to redistribute to lists, requires prior specific permission and/or a fee. Request permissions from permissions@ acm.org.

SoICT 2015, December 03 - 04, 2015, Hue City, Viet Nam

(C) 2015 Copyright held by the owner/author(s). Publication rights licensed to ACM. ISBN 978-1-4503-3843-1/15/12 . .\$15.00

DOI: http://dx.doi.org/10.1145/2833258.2833267 world data it has several benefits that make it suitable for large-scale experimentation:

- It allows for controlled experiments, which means that the researcher can focus on changes with respect to specific characteristics while leaving others untouched. This is akin to performing controlled experiments in medical sciences, psychology and sociology.

- The ground truth is known, which means that we can make a qualitative assessment on whether learning algorithms can retrieve the underlying concepts.

- It is available in abundance without legal, ethical and commercial restrictions, which means that it can be freely shared among researchers and as such improves the reproducibility of results.

As a result, artificial data is often used to determine relationships between data characteristics and algorithm performance $[33,37]$ and to analyze the behavior of algorithms in changing environments [23, 29, 30, 35].

Traditional approaches to data generation are often tailored to a specific type of data or concept class, for example, decision trees [8], rules [19], clusters [32], sequences [1, $27,40]$ or regression functions $[11,23]$. Even the more general purpose approaches $[10,33]$ are typically limited in that they only allow control of the generation process through high level parameters (e.g. number of attributes, number of classes).

In this paper, we develop a new methodology for data generation. Our framework follows a declarative approach based on probabilistic logic programming. It has several advantages over existing work:

- It is declarative, that is, the user only needs to specify distributions on variables and relations between them. The data generation process is completely handled by the underlying engine. This also increases transparency and reproducibility because all the properties of the data are fully described in a relatively simple model.

- It is flexible and general. The framework is based on Prolog [24, 39], which is a general purpose programming language. It is therefore capable of describing arbitrarily complex distributions and structures.

- It is modular. It allows different components to be defined separately and be reused. 
- It is based on existing technology, namely Prolog and ProbLog [7, 9], which means that there are many resources available that describe how to program these models.

This paper is organized as follows. In Section 2 we give an overview of the language of ProbLog, we discuss how we deal with continuous distributions and how the system generates examples. In Section 3 we show how we can use this language to describe complex distributions for data generation. Section 4 gives an overview of related work and Section 5 concludes this paper.

\section{LANGUAGE}

\subsection{Prolog}

Prolog [24] is a general purpose programming language based on first-order logic. Unlike many other programming languages, Prolog is declarative, that is, it follows the principle of algorithm $=$ logic + control where only the logic is defined by the user and control is defined entirely by the Prolog engine.

A Prolog program consists of a list of clauses. Each clause is of the form

head :- body $1, \ldots$, bodyN .

where head, body $1, \ldots$, body $N$ are terms. A term consists of a functor followed by a list of arguments: term $(\arg 1, \arg 2, \ldots)$. The arguments can be constants, variables (starting with an uppercase letter) or other terms. A clause signifies that when all the terms in the body are true, then the head is true. A fact is a clause with body true and in this case the body is omitted. A program can contain multiple clauses with the same head. Such clauses indicate a logical disjunction.

Consider the following example describing a partial family tree.

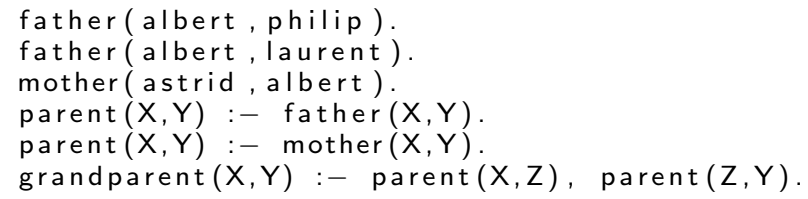

It contains three facts stating that Albert is the father of Philip and Laurent, and that Astrid is the mother of Albert. It also contains three rules defining the concepts of 'parent' and 'grandparent'. The uppercase identifiers $X, Y$ and $Z$ are variables.

Execution of a Prolog program starts from a query. For example, we can ask whether Albert is a parent of Astrid (? - parent(albert, astrid )) or we can ask who the grandchildren of Astrid are (? - grandparent(astrid ,X)). The first query will return false, and the second will return all assignments to the variables in the query that satisfy the model $(\{X /$ philip $\}$ and $\{\mathrm{X} /$ laurent $\})$. The evaluation of such queries uses SLDresolution. When proving a query, Prolog selects clauses that match the query from top-to-bottom and recursively queries the body literals from left-to-right. A trace is shown in Figure 1.

\subsection{ProbLog}

ProbLog [7, 9] extends Prolog with probabilities, that is, each clause is annotated with a probability of being true.
For example, we can introduce some uncertainty in the family relations of the earlier example.

$0.99:$ : father (albert, philip).

$0.80:$ : father (albert, laurent).

mother(astrid, albert).

In this case, we say that there is a $1 \%$ probability that Albert is not the father of Philip, and a $20 \%$ probability that he is not the father of Laurent. We are still $100 \%$ sure that Astrid is the mother of Albert, so we can omit the probability.

The primary task of ProbLog is to answer the same type of queries as Prolog, but instead of giving a deterministic answer, ProbLog will compute the probability that the query is true. ProbLog uses possible world semantics where each possible world is a deterministic assignment of the probabilistic facts. The probability of such a world is the product of all the corresponding assignments of the facts. The probability of a query is then defined as the sum of the probabilities of all possible worlds in which the query is true. This definition has two important properties: (1) all probabilistic facts are independent and (2) each probabilistic fact has a unique truth value within the same possible world. Efficiently computing this probability is a non-trivial problem that is tackled using knowledge compilation techniques [9].

In this paper, however, we focus on a different task, namely sampling from a ProbLog model. Sampling from a ProbLog model is relatively straightforward and closely resembles the SLD-resolution of Prolog. When a probabilistic fact is encountered, we simply make a choice indicating whether it becomes true or false, depending on the probability. We also record the decision made, such that if the fact gets reused, it receives the same truth value. Earlier versions of ProbLog supported this sampling procedure for inference.

From a theoretical viewpoint we may assume that only facts are annotated with probabilities. ProbLog provides additional syntax to support probabilities on rules and mutually exclusive choices.

A probabilistic rule $\mathrm{p}:$ : head(VH) :- body(VB) can be rewritten as the combination of a non-probabilistic rule and a probabilistic fact.

$\mathrm{p}:$ : rule $1(\mathrm{VH}, \mathrm{VB})$.

head $(\mathrm{VH}):-\operatorname{body}(\mathrm{VB}), \operatorname{rule} 1(\mathrm{VH}, \mathrm{VB})$.

The probabilistic fact is defined using all the variables that occur in the clause, which means that different groundings of the body lead to independent choices.

Mutually exclusive choices can be specified using an annotated disjunction [38] such as

$0.5:: \operatorname{coin}(\mathrm{C}$, heads $) ; 0.5:: \operatorname{coin}(\mathrm{C}, \mathrm{tails})$.

which encodes that coin $C$ can either take the value "heads", or the value "tails", but not both. When the probabilities of the disjuncts do not sum to one, it is also possible that neither of the options is taken. This construct can also be used in the head of a rule. Such a statement can be rewritten in terms of probabilistic facts and deterministic rules by introducing a fact for each possible choice with an adjusted weight.

An important observation to make is that, according to ProbLog's semantics, each occurrence of the same ground probabilistic atom in the same sample takes the same value. For example, in the model

$0.5:: \operatorname{coin}(\mathrm{h}) ; 0.5:: \operatorname{coin}(\mathrm{t})$.

two_coins $(X, Y):-\operatorname{coin}(X), \operatorname{coin}(Y)$. 


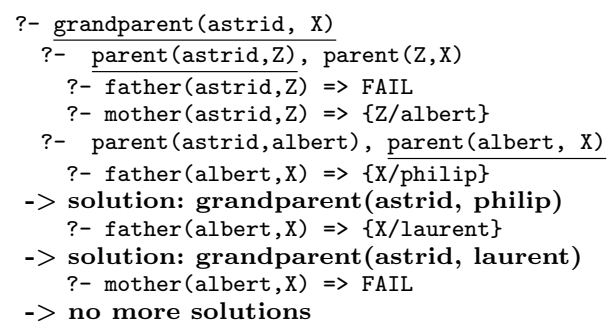

Figure 1: Prolog trace. By default Prolog only returns one solution and asks the user whether it should produce more.

query (two_coins $\left.\left(_{-},-\right)\right)$.

only the examples two_coins $(\mathrm{h}, \mathrm{h})$ and two_coins $(\mathrm{t}, \mathrm{t})$ are possible because both $\operatorname{coin}(X)$ and $\operatorname{coin}(Y)$ refer to the same ground facts. In order to have multiple, independent choices we should add an additional unique identifier

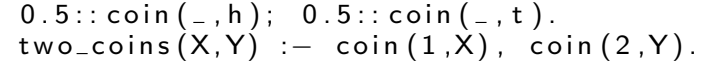

ProbLog programs also allow users to specify evidence, that is, to specify atoms that are known to be true or false. In a sampling setting, these can be used to only generate samples that satisfy certain conditions. In the remainder of this paper, we will not make use of this functionality so we omit the details.

\subsection{Extension to continuous distributions}

Standard ProbLog is limited to discrete probabilities. A realistic data generator should however also support continuous distributions.

The concept of ProbLog with continuous distributions was first introduced by Gutmann et al [15, 17]. We follow the same semantics but we use a simplified syntax that is closer to the original ProbLog syntax. The language specification of ProbLog allows arbitrary terms as probabilities. We can use this to specify continuous distributions on facts (and rules). For example, we can specify that the height of person $\mathrm{X}$ is normally distributed as

normal $(170,6):$ : height $(X)$.

We support a wide range of distributions (normal, Poisson, exponential, beta, gamma, uniform, triangular, ...).

In Prolog we can assign a boolean value to a term by querying it in the program. The introduction of continuously distributed variables, means that we also introduce a new concept: a term-with-a-value or vterm. A vterm is a term that appears in the head of a clause annotated with a continuous distribution. Such a term does not support logical operations with other vterms such as 'and', 'or' and 'not', which means that clauses defining the same term should be mutually exclusive and the body of a clause should contain at most one vterm.

We can convert a vterm to a regular Prolog term and its value using the builtin value(+VTerm, - Value). This builtin queries for the given vterm and assigns its value to the output argument Value. As with regular Prolog queries, this call can fail or produce multiple results (in case of a non-ground

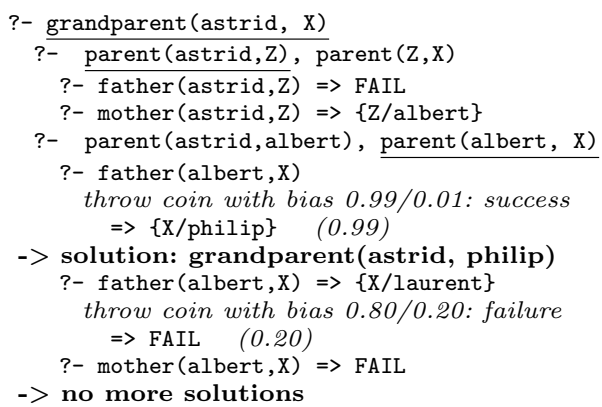

Figure 2: ProbLog sampling trace. Two probabilistic choices are made. The probability of the produced solution set is $0.99 \times 0.20=0.198$.

vterm). The obtained value is a regular Prolog atom (e.g. representing a number) that can be used in, for example, Prolog arithmetic expressions.

To attach a value to a Prolog term, we use the fixed (Value) annotation. These constructs are demonstrated in the following model.

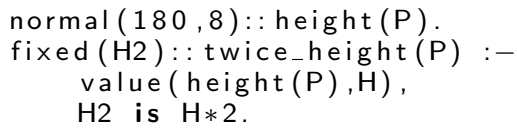

The evaluation of models with continuous distributions is the same as for discrete probabilities. Upon encountering a continuously distributed fact, we sample a value from its distribution and record that value.

\section{DATA GENERATION}

In the previous section we discussed the basic structure of the language. We now demonstrate how we can use these models to generate data. In this section we show a number of building blocks that can be combined to make complex data generators.

\subsection{Basic data generation}

First, let us focus on the simplest data generation task, that is, generate a tuple of attribute values. Consider the following model which randomly generate a personal record containing a (Dutch) person's gender and their height.

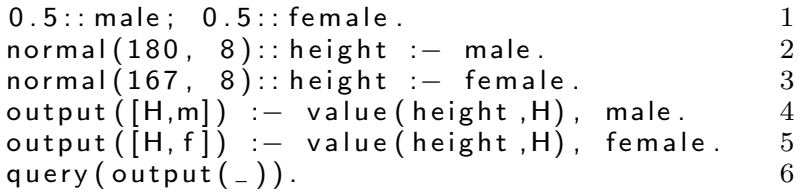

Line 1 generates a binary attribute representing the gender of the person where each gender has $50 \%$ probability. On Lines $2-3$ we then generate the height of the person based on their gender where we indicate that this height is distributed according to a normal distribution. Lines $4-5$ then define the structure of the output record, and Line 6 indicates which predicate specifies the output. The output produced by sampling from this model is shown in Figure 3.

By using these simple relations we can build very complex distributions. 
output $([164.220750786, \mathrm{f}])$
output $([166.120265276, \mathrm{f}])$.
output $([177.341443765, \mathrm{~m}])$.
output $([185.649079212, \mathrm{~m}])$.
output $([178.894743264, \mathrm{~m}])$.
output $([176.937380250, \mathrm{~m}])$.
output $([158.758450415, \mathrm{f}])$.
output $([184.218990138, \mathrm{~m}])$.
output $([183.145639384, \mathrm{~m}])$.
output $([175.308625610, \mathrm{f}])$.

(a) First 10 results.

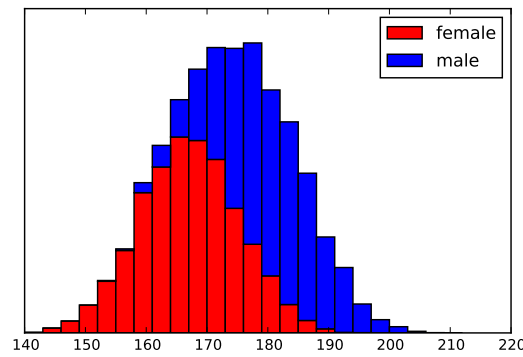

(b) Distribution of 10000 samples.

Figure 3: Output for "Height of people" model of Section 3.1.

\subsection{Noise}

Realistic data often does not exactly follow a logical model because there may be errors in measurements or other random factors that are not described in the model. Such effects are typically simulated by introducing noise on the data.

Noise can occur in two ways: (1) by random variations in the value of a numeric attribute, or (2) by random flips in the value of a nominal attribute. The first type of noise is demonstrated in the following model.

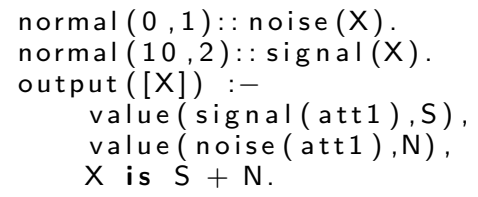

Here we take a simple additive Gaussian noise model, but more complex definitions can be imagined.

The second model demonstrates the occurrence of noise on a binary attribute.

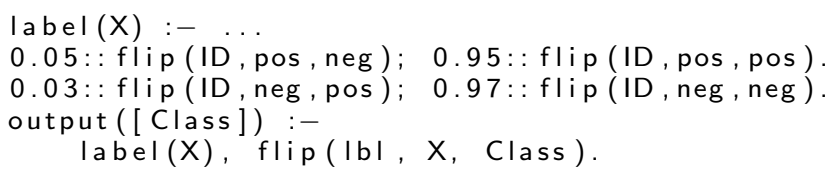

Here we randomly flip the class label from positive to negative with $5 \%$ probability or from negative to positive with $3 \%$ probability. Note that both noise models can be specified independently of the rest of the model.

\subsection{Missing values}

Another important aspect of realistic data is that sometimes not all values are known. The following model demonstrates how missing values can be introduced.

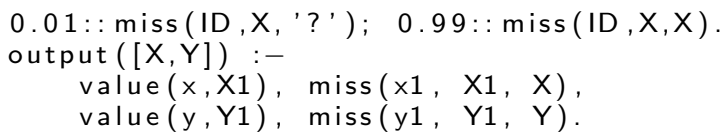

We assume that the predicates $x$ and $y$ are defined elsewhere. This model specifies that the value of an attribute is removed with $1 \%$ probability.

\subsection{Structured output - sequences}

The previous examples showed how to generate rows of attribute values. The generation process also allows the generation of structured data.
For example, the following program generates sequences of arbitrary length consisting of the characters $\{a, b, c\}$.

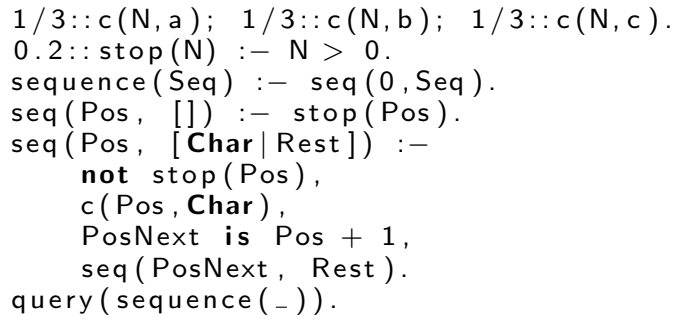

The sequences are governed by two distributions: the distribution of the characters (Line 1) and the distribution of the length of the sequence (encoded as a probability to stop, on Line 2). To avoid empty sequences, we add the condition $\mathrm{N}>0$ as part of the stopping criterion. The sequence is represented as a Prolog list that is built by the seq/2 predicate. This predicate is defined using two clauses in the typical Prolog style. On Line 4 we have the termination clause, which is used when the decision is made to stop. This clause returns an empty sequence. Lines 5-9 defines the case when the decision is made not to stop. The test on Line 6 ensures that both clauses are mutually exclusive. In this case, we select a character (Line 7) and we prepend it to the rest of the sequence (still to be generated) using the expression [Char|Rest] in the head of the clause on Line 5. The remainder of the sequence Rest is generated by a recursive call on Line 9. It is important to note that this model does not ensure termination, and is hence capable of generating sequences of arbitrary length.

The model above is very simple and can be extended in multiple ways, for example, by including a Markov chain by basing the choice of character on the previous character(s).

\subsection{Concept generation}

The ability to generate structured output also makes it possible to generate arbitrary mathematical functions, decision trees and other data structures that are typically used to represent concepts in machine learning.

The following model generates a decision tree where each internal node contains a test Feat $<$ Val, and each leaf contains a class label.

$0.6:$ : is_le a f (ID).

$0.5:$ : is_class (ID , 0 ); $0.5::$ is_class (ID , 1$)$.

uniform $(A, B)$ : : in_range (_, A, B ).

simpletree(IDin, IDout, class(C)) :- 


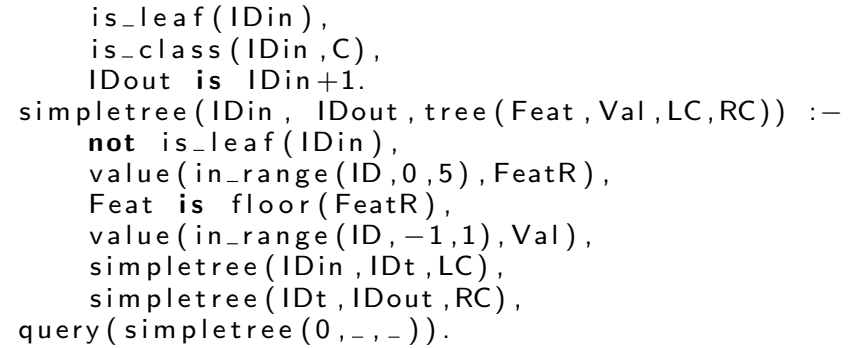

For space reasons, this model is severely simplified because it does not take into account the distribution of class labels or whether the tests on the same branch are a feasible combination.

\subsection{Generating sequential data}

Until now, we assumed that the examples generated by the generator are independent and drawn from the same distribution (i.i.d. assumption). In many applications this is not the case, for example, in time series data or in data streams with concept drift. Although the distribution of such cases can be modeled using the constructs above by modeling multiple examples in the same model, we choose to take a different approach that is more scalable.

By introducing the builtin previous(Query, Default) we allow access to information from the previously generated example. In this way we can carry over information from one example to the next. A simple case is when we want to assign a sequential number to all examples.

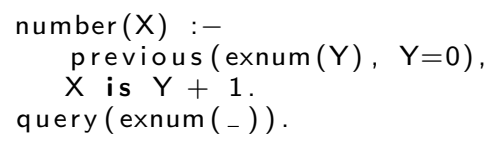

The call to previous has access to the output of the previously generated example. This means that the requested predicate should be listed as a query of the model. If there is no previous example, the default action is performed (in this case initialize the number to zero).

Because the arguments of the predicate can contain arbitrary Prolog terms, the information shared between examples can be arbitrarily complex. This paradigm is thus not restricted to first-order Markov Chains. Consider for example the following model that stores all previously generated values.

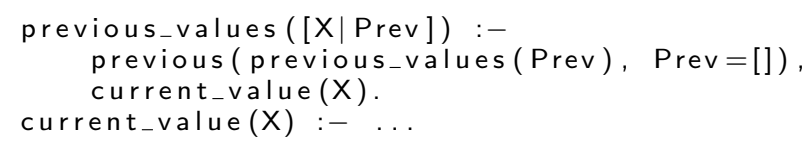

Clearly, when using this functionality we can no longer guarantee constant time and memory usage for each example generated.

One important use case for this functionality is when we want to generate a concept and, at the same time, data labeled according to that concept. This can be achieved by adding the following statements to the model.

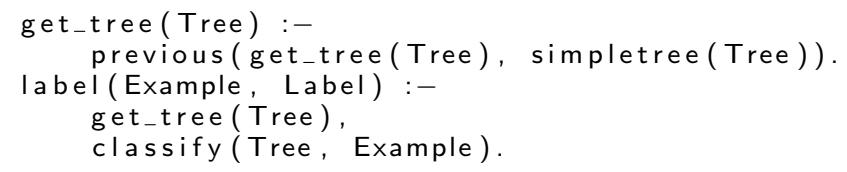

where simpletree is defined in Section 3.5. In the first example, the predicate simpletree is called to generate a ran- dom decision tree. In subsequent examples the same tree is reused.

We can also use this functionality to generate the parameters of our model itself.

\subsection{Concept drift}

One of the areas where the use of data generation is popular is the study of concept drift. In this area it is very important to have control over changes in the data over time in order to verify the ability of algorithms to cope with such changes. Consider for example the popular STAGGER benchmark [34]. The data consists of 120 geometrical figures that have three properties (size, color and shape). They are labeled according to a given concept which is changed after every 40 examples.

The full specification of this benchmark is shown in the following model.

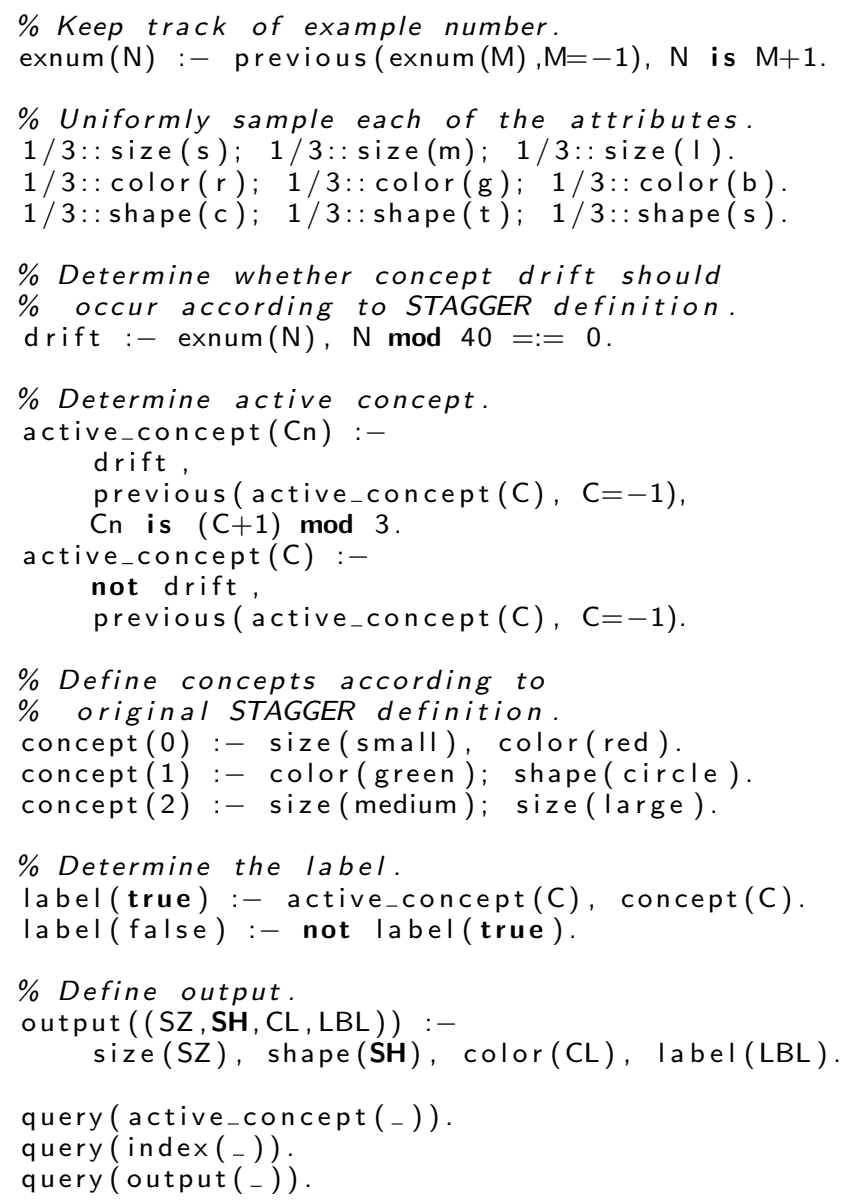

In the STAGGER benchmark, the occurrence of concept drift is hardcoded. Using our framework it is also possible to make this a soft decision using probabilistic facts. For example, we can specify that concept drift occurs on average every 40 examples according to a Poisson distributed interval.

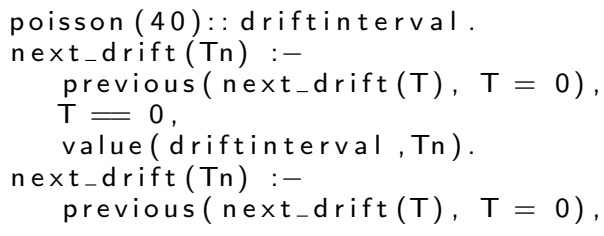


$\mathrm{T} \backslash==0, \operatorname{Tn}$ is $\mathrm{T}-1$.

drift :- previous (next_drift $(\mathrm{T}), \mathrm{T}=0), \mathrm{T}=0$.

The predicate next_drift $(\mathrm{T})$ represents a counter that counts down to the next concept drift occurrence. When it reaches zero a concept drift occurs and the counter is reset using the Poisson distribution. In a similar way, we can introduce gradual drift, where only a gradually increasing proportion of examples is labeled by the new concept.

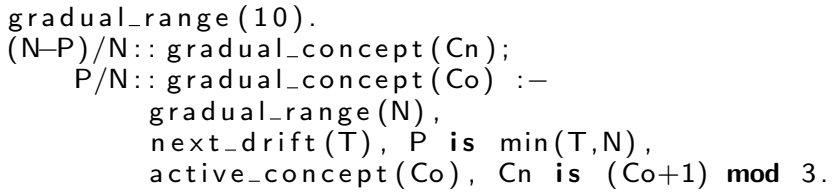

This model specifies that over a window of 10 examples before the drift point, the new concept can already appear with a probability increasing with $10 \%$ for each example.

\subsection{Other examples}

Apart from the examples mentioned above, this system has been tested on more elaborate models. All of the following models are available from the author's website ${ }^{1}$.

Decision tree Generates random decision trees (tree).

Molecules Generates structurally correct molecules (tree).

Bongard problems Generates random Bongard problems, a common benchmark for inductive logic programming systems [5] (logical interpretation).

Michalski trains Generates a dataset of random train descriptions, another well known dataset from ILP [26] (logical interpretation).

Lorum ipsum Generates random text paragraphs (text).

The sampling algorithm itself has been integrated into ProbLog and is part of the standard ProbLog distribution ${ }^{2}$.

\section{RELATED WORK}

Many results in machine learning have been (partially) validated based on artificially generated data. The data generation process is usually only marginally described as part of the experimentation section. In some cases, however, such data generators have become de-facto benchmarks in certain areas.

The LED and waveform generators [2] are two of the earliest artificial datasets described in literature. They were introduced to evaluate classification and regression trees.

There is particular interest in data generation from the field of data stream analysis and the related research on concept drift. One of the oldest benchmarks in this area is STAGGER [34] which consists of geometric objects described by size, shape and color. The generator switches between three different concepts for labeling these objects. Another standard benchmark is the SEA dataset [35] where three features are generated uniformly, and the data point is labeled using a linear function with a changing threshold and noise level. As part of the evaluation for their VFDT algorithm, Domingos \& Hulten [8] created a random tree generator. This generator first constructs a random decision tree,

\footnotetext{
${ }^{1}$ http://people.cs.kuleuven.be/ anton.dries/datagen

${ }^{2}$ https://dtai.cs.kuleuven.be/problog
}

and then uses it to label randomly generated instances. In their follow-up work on handling concept drift [20], the same authors introduced the concept of rotating hyperplanes as an elegant way of generating time-changing data. The generated data is labeled based on hyperplanes whose orientation and position is varied smoothly over time. In a similar fashion, Kirby [23] proposed a generator for time-changing data based on randomly generated radial-basis functions. Concept drift can be introduced by changing the position of their centroids over time. Minku et al. [29] introduce several additional benchmarks based on circles, sine waves and some generalizations of the STAGGER concepts.

In traditional machine learning, the Autouniv artificial model generator [19] produces a rule-based class model (in Prolog) together with a joint distribution of the description attributes. The generation process is directed by a number of high-level parameters (e.g. the number of informative attributes, the number of noise attributes, number of attributes part of the same factor, ...). It only support discrete attributes and offers only limited control over the logical structure of the produced model.

Friedman [11] introduces a random function generator in the context of function approximation. These functions are commonly used in the study of regression techniques.

Van Der Walt et al. [37] used datasets generated using simple Gaussian mixture models for debunking common myths in machine learning. Their work demonstrates the usefulness of artificially generated data.

In the field of sequence mining, the Almaden Quest generator [1] is well-known. This generator can be used to generate datasets for sequence mining or association mining that mimics a customer-sales environment. The properties of the data can be specified using high-level parameters such as number of customers, number of transactions per customer, number of items per transaction and length of patterns to introduce. In the related field of episode mining, Laxman et al. [27] propose an approach based on hidden Markov models (HMM). They study "Episode Generating HMMs" and how they can be used to model episode mining problems. Zimmermann [40] observed several shortcomings with their approach such as the lack of timestamps, the assumption of uniformly distributed events, and the lack of realistic distributions such as normal and Poisson distributions.

Rachkovskij \& Kussul [33] propose a more general algorithm that generates samples from a partitioned feature space. Their method offers parameters for partitioning the feature space into different regions ("classes") as well as for the distribution of the samples generated from each class.

The work by Pei \& Zaïane [32] focusses on generating data for unsupervised learning and outlier detection. The parameters include the number of clusters, distance between clusters, and complexity of the clusters. The generator produces two-dimensional data containing clusters of varying complexity.

Most generators work by fixing the distributions of the base facts, or by setting a few high level parameters. Frasch et al. [10] instead try to fix distributional parameters of the output. Their WGKS generator focusses on representing very simple classification models and they focus on controlling the Bayes error between class densities. Their generator is tailored to this particular property and it is hard to extend to more general properties.

Many of the generators mentioned above are hardcoded to 
produce a certain type of concept, offer only high-level parameters to direct the generation process (e.g. number of attributes, number of classes, ...) or have hidden assumptions about, for example, distribution of noise. This is in contrast with the declarative approach described in this paper, where every aspect of the generation process is explicitly described in the model. Two approaches follow a more declarative approach. Jeske et al. [21] generate social data based on a semantic graph specified by the user. Haddawy [18] generates Bayesian networks based on a simple description in logic. They do not support the type of structured output presented in this paper.

In the probabilistic programming community there are several languages for describing distributions. For example, Church [14] is a probabilistic programming language designed for the description of generative models. However, unlike ProbLog, Church is not based on first-order logic which makes it more cumbersome to encode logical relations. ProbLog's logical nature also makes it very suitable for learning itself $[6,16,22]$.

The constraint programming community also offers different languages that describe sets of solutions in a declarative manner. For example, MiniZinc [31] is a language for specifying constraint satisfaction problems. In MiniZinc one can describe possible examples by means of constraints that they should satisfy. However, sampling from such models in an unbiased way is a non-trivial task [13].

In the database community data generation tools (such as TCP-H [36]) are used to automatically generate benchmark datasets. Although, this tool generates large and complex databases, the underlying assumptions are rather simple and the database schema is fixed. The work by Bruno and Chaudhuri addresses these issues by proposing a specialpurpose data generation language based on database principles [3].

The need for artificial data is complementary to the need for real data. One problem that this paper addresses is the need to share data for reproducibility. In that sense this is related to the work on building repositories of data such as UCI [28], UCR [4] and FIMI [12]. Note that many of the datasets in these repositories are artificial.

\section{CONCLUSION}

In this paper we present a declarative framework for data generation based on probabilistic logic programming. This framework builds on the general purpose programming language Prolog extended with probabilities and continuous distributions $[7,9]$. The combination of logic programming and probabilistic models allows us to model a wide variety of non-trivial data generation tasks. Our framework support noise, missing values, concept drift, structured data and much more. It can be used to generate concepts as well as complete data sets and streams of data.

For future work, there are still a few issues that require further research. Currently, all the models presented here are written in a generative way. Using ProbLog's evidence keyword it is also possible to add high-level constraints that are hard to encode in a generative model. Currently, such constraints are handled using rejection sampling which can be quite slow. It would be interesting to see how such constraints can be propagated into the sampling process. This is also of interest for other ProbLog related tasks such as inference.
The syntax outlined above only works for specifying distributions on single variables. This makes it cumbersome to specify multi-varied distributions.

An important requirement of artificially generated data is that it is realistic. It would therefore be interesting to learn generative models from example data. This is useful when the original dataset is too small or when the data itself can not be shared because of privacy issues or a non-disclosure agreement. The choice of ProbLog as the language should make this more feasible given that it lies on the intersection of probabilistic programming and statistical relational learning and quite a few learning problems have already been investigated $[6,16,22]$.

\section{ACKNOWLEDGEMENTS}

This project was funded by NAFOSTED and FWO under grant number FWO.102.2013.04 "Prediction-based optimization for dynamic transport scheduling". The author thanks Angelika Kimmig and Wannes Meert for their constructive comments.

\section{REFERENCES}

[1] R. Agrawal and R. Srikant. Mining sequential patterns. In International Conference on Data Engineering (ICDE), pages 3-14, Washington, DC, USA, 1995. IEEE Computer Society.

[2] L. Breiman, J. Friedman, C. J. Stone, and R. A. Olshen. Classification and regression trees. CRC press, 1984.

[3] N. Bruno and S. Chaudhuri. Flexible database generators. In International Conference on Very Large Data Bases (VLDB), pages 1097-1107. VLDB Endowment, 2005.

[4] Y. Chen, E. Keogh, B. Hu, N. Begum, A. Bagnall, A. Mueen, and G. Batista. The UCR time series classification archive.

http://www.cs.ucr.edu/ eamonn/time_series_data/, July 2015.

[5] L. De Raedt. Inductive logic programming. In Encyclopedia of Machine Learning, pages 529-537. Springer US, 2010.

[6] L. De Raedt, A. Dries, I. Thon, G. Van den Broeck, and M. Verbeke. Inducing probabilistic relational rules from probabilistic examples. In International Joint Conference on Artificial Intelligence (IJCAI), 2015.

[7] L. De Raedt, A. Kimmig, and H. Toivonen. Problog: A probabilistic prolog and its application in link discovery. In International Joint Conference on Artificial Intelligence (IJCAI), volume 7, pages 2462-2467, 2007.

[8] P. Domingos and G. Hulten. Mining high-speed data streams. In ACM SIGKDD international conference on Knowledge Discovery and Data Mining (KDD), pages 71-80. ACM, 2000.

[9] D. Fierens, G. Van den Broeck, J. Renkens, D. Shterionov, B. Gutmann, I. Thon, G. Janssens, and L. De Raedt. Inference and learning in probabilistic logic programs using weighted Boolean formulas. Theory and Practice of Logic Programming, 15:358-401, 52015.

[10] J. V. Frasch, A. Lodwich, F. Shafait, and T. M. Breuel. A Bayes-true data generator for evaluation of 
supervised and unsupervised learning methods. Pattern Recognition Letters, 32(11):1523-1531, Aug. 2011.

[11] J. H. Friedman. Greedy function approximation: a gradient boosting machine. Technical report, Dept. of Statistics, Stanford University, 1999.

[12] B. Goethals. Frequent itemset mining dataset repository. http://fimi.ua.ac.be/data/, 2003.

[13] V. Gogate and R. Dechter. A new algorithm for sampling csp solutions uniformly at random. In International Conference of Principles and Practice of Constraint Programming (CP), volume 4204 of $L N C S$, pages 711-715. Springer Berlin Heidelberg, 2006.

[14] N. D. Goodman, V. K. Mansinghka, D. M. Roy, K. Bonawitz, and J. B. Tenenbaum. Church: A language for generative models. In International Conference on Uncertainty in Artificial Intelligence (UAI), pages 220-229, 2008.

[15] B. Gutmann, M. Jaeger, and L. De Raedt. Extending problog with continuous distributions. In International Conference on Inductive Logic Programming (ILP), volume 6489 of $L N C S$, pages 76-91. Springer Berlin Heidelberg, 2011.

[16] B. Gutmann, A. Kimmig, K. Kersting, and L. De Raedt. Parameter learning in probabilistic databases: A least squares approach. In European Conference on Machine Learning and Principles and Practice of Knowledge Discovery in Databases (ECMLPKDD), volume 5211 of $L N C S$, pages 473-488. Springer Berlin Heidelberg, 2008.

[17] B. Gutmann, I. Thon, A. Kimmig, M. Bruynooghe, and L. De Raedt. The magic of logical inference in probabilistic programming. Theory and Practice of Logic Programming, 11(4-5):663-680, 2011.

[18] P. Haddawy. Generating bayesian networks from probability logic knowledge bases. In International Conference on Uncertainty in Artificial Intelligence (UAI), pages 262-269, San Francisco, CA, USA, 1994. Morgan Kaufmann Publishers Inc.

[19] R. J. Hickey. Structure and majority classes in decision tree learning. Journal of Machine Learning Research, 8:1747-1768, Dec. 2007.

[20] G. Hulten, L. Spencer, and P. Domingos. Mining time-changing data streams. In ACM SIGKDD international conference on Knowledge Discovery and Data Mining (KDD), pages 97-106. ACM, 2001.

[21] D. R. Jeske, B. Samadi, P. J. Lin, L. Ye, S. Cox, R. Xiao, T. Younglove, M. Ly, D. Holt, and R. Rich. Generation of synthetic data sets for evaluating the accuracy of knowledge discovery systems. In $A C M$ SIGKDD international conference on Knowledge Discovery and Data Mining (KDD), pages 756-762. ACM, 2005.

[22] A. Kimmig, L. De Raedt, and H. Toivonen. Probabilistic explanation based learning. In European Conference on Machine Learning and Principles and Practice of Knowledge Discovery in Databases (ECMLPKDD), volume 4701 of $L N C S$, pages $176-187$. Springer Berlin Heidelberg, 2007.

[23] R. B. Kirkby. Improving hoeffding trees. PhD thesis, The University of Waikato, 2007.

[24] R. Kowalski. Logic for problem solving. Elsevier
Science Inc., 1979.

[25] P. Langley. Machine learning as an experimental science. Machine Learning, 3(1):5-8, 1988.

[26] J. Larson and R. S. Michalski. Inductive inference of vl decision rules. ACM SIGART Bulletin, (63):38-44, 1977.

[27] S. Laxman, P. Sastry, and K. Unnikrishnan. Discovering frequent episodes and learning hidden markov models: A formal connection. IEEE Transactions on Knowledge and Data Engineering, 17(11):1505-1517, 2005.

[28] M. Lichman. UCI machine learning repository. http://archive.ics.uci.edu/ml, 2013.

[29] L. Minku, A. White, and X. Yao. The impact of diversity on online ensemble learning in the presence of concept drift. IEEE Transactions on Knowledge and Data Engineering, 22(5):730-742, May 2010.

[30] A. Narasimhamurthy and L. I. Kuncheva. A framework for generating data to simulate changing environments. In International Multi-Conference on Artificial Intelligence and Applications (ICAIA), 2007.

[31] N. Nethercote, P. J. Stuckey, R. Becket, S. Brand, G. J. Duck, and G. Tack. Minizinc: Towards a standard cp modelling language. In International Conference of Principles and Practice of Constraint Programming (CP), pages 529-543. Springer, 2007.

[32] Y. Pei and O. Zaïane. A synthetic data generator for clustering and outlier analysis. Technical report, University of Alberta, Edmonton, CA, 2006.

[33] D. A. Rachkovskij and E. M. Kussul. Datagen: a generator of datasets for evaluation of classification algorithms. Pattern Recognition Letters, 19(7):537 544, 1998.

[34] J. Schlimmer and R. Granger. Incremental learning from noisy data. Machine Learning, 1(3):317-354, 1986.

[35] W. Street and Y. Kim. A streaming ensemble algorithm (sea) for large-scale classification. In $A C M$ SIGKDD international conference on Knowledge Discovery and Data Mining (KDD), pages 377-382, 2001.

[36] Transaction Processing Performance Council (TPC). TPC benchmark $\mathrm{H}$. http://www.tpc.org/tpch/spec/tpch2.1.0.pdf, 2003.

[37] C. Van Der Walt and E. Barnard. Data characteristics that determine classifier performance. SAIEE Africa Research Journal, 98(3):87-93, 2006.

[38] J. Vennekens, S. Verbaeten, and M. Bruynooghe. Logic programs with annotated disjunctions. In International Conference on Logic Programming (ICLP), pages 431-445. Springer, 2004.

[39] D. H. D. Warren, L. M. Pereira, and F. Pereira. Prolog - the language and its implementation compared with lisp. In Symposium on Artificial Intelligence and Programming Languages, pages 109-115, New York, NY, USA, 1977. ACM.

[40] A. Zimmermann. Generating diverse realistic data sets for episode mining. In International Conference on Data Mining Workshops (ICDMw), pages 611-618, Dec 2012. 